\title{
Residue Calculus and Applications
}

\author{
By \\ Mohamed ELKADI* and Alain YGER**
}

\begin{abstract}
We present a new algorithm in order to compute the multidimensional residue of a polynomial map based on a perturbation argument and the Generalized Transformation Law. Then we use it for studying some fundamental problems in Computer Aided Geometric Design.
\end{abstract}

\section{$\S 1$. Introduction}

Let $F=\left(F_{1}, \ldots, F_{n}\right)$ be a polynomial map in the ring $\mathbb{C}[x]:=\mathbb{C}\left[x_{1}, \ldots, x_{n}\right]$ such that the algebraic affine variety $\left\{\alpha \in \mathbb{C}^{n}: F_{1}(\alpha)=\cdots=F_{n}(\alpha)=0\right\}$ is non-empty and finite. The multidimensional residue of $F$, denoted by $\operatorname{Res}_{F}$, plays a central role in several areas of mathematics : Complex Analysis, Analytic Geometry, Algebraic Geometry, Computational Algebra, Complexity theory (see $[1,4,5,17,18,15,23,3])$; so the problem of finding efficient algorithms for the computation of the residue is an important task. When the system of polynomials $F_{1}, \ldots, F_{n}$ have no common zeroes at infinity the computation of $\operatorname{Res}_{F}$ is easily reduced to the univariate case. In the general setting, resultants [31], Gröbner bases [12], bezoutian matrices [20], can be used to compute the multidimensional residue. But such methods are not fully satisfactory from a computational point of view since the sizes of the intermediate data do not fit with the bounds predicted in the arithmetic Bézout problem by [6, 25]. We

\footnotetext{
Communicated by T. Kawai. Received January 19, 2005. Revised October 28, 2005. 2000 Mathematics Subject Classification(s): 32A27, 68W30, 65D17.

Key words: Multidimensional residue, Transformation laws, Residue calculus, Implicitization Problem.

*Université de Nice Sophia Antipolis, Parc Valrose, 06108 Nice Cedex2, France. e-mail: elkadi@math.unice.fr

** LaBaG, Université Bordeaux 1, Cours de la Libération, 33405 Talence, France. e-mail: yger@math.u-bordeaux.fr
} 
present here a new and simple algorithm to compute effectively $\operatorname{Res}_{F}$. It is inspired by the so-called Arnold's perturbation method (see for example [2]). The strategy is to reduce the computations to the case of a perturbed polynomial map without common zeroes at infinity, and to deduce algebraic relations between the components of this map and the coordinate functions $x_{1}, \ldots, x_{n}$. Then we use a generalized version of the Transformation Law proved in [6] to obtain $\operatorname{Res}_{F}$ (see Subsection 2.2). A similar approach was already followed in [20]. However here, instead of computing symbolic determinants, we rely on constant coefficients of some Laurent series. The obtained algorithm is then applied to solve important questions in CAGD (Computer Aided Geometric Design), namely the Implicitization Problem, the determination of the Singular locus and the Intersection Problem for rational hypersurfaces (see [29], [7], [11], [13], [14], [16], [10]).

The paper is organized as follows. Section 2 recalls important facts about multidimensional residue theory and shows that the global multidimensional residue of a polynomial map $F$ can be computed via a perturbed system $\Phi(\lambda, u ;$.$) without zeroes at infinity, using the Generalized Transformation Law.$ Section 3 studies problems in CAGD and solve them by the method developed in section 2 for the computation of residues. At the end we give some examples in order to illustrate our approach.

Here are some notations that will be used hereafter : $\mathbb{K}$ denotes an infinite subfield of $\mathbb{C}, \mathbb{K}[x]=\mathbb{K}\left[x_{1}, \ldots, x_{n}\right]$ is the ring of polynomials in the variables $x_{1}, \ldots, x_{n}$ with coefficients in $\mathbb{K}, \mathbb{K}(x)$ is its fraction field, $\mathbb{K}\left[x_{0}, x\right]$ is the ring $\mathbb{K}\left[x_{0}, \ldots, x_{n}\right]$, and the dimension of a $\mathbb{K}$-vector space $\mathrm{E}$ is denoted by $\operatorname{dim}_{\mathbb{K}} E ;$ if $m=\left(m_{1}, \ldots, m_{n}\right) \in \mathbb{N}^{n}$, we set $|m|=m_{1}+\cdots+m_{n}$ and $x^{m}=x_{1}^{m_{1}} \ldots x_{n}^{m_{n}}$; given $G_{1}, \ldots, G_{l} \in \mathbb{K}[x], \mathcal{Z}\left(G_{1}, \ldots, G_{l}\right)$ will denote the affine algebraic variety

$$
\mathcal{Z}\left(G_{1}, \ldots, G_{l}\right)=\left\{\alpha \in \mathbb{C}^{n}: G_{1}(\alpha)=\cdots=G_{l}(\alpha)=0\right\} ;
$$

the Jacobian determinant of a polynomial map $F=\left(F_{1}, \ldots, F_{n}\right)$ will be denoted as $\operatorname{Jac}(F)$. The algebraic closure of a field $\mathbb{L}$ will be denoted by $\overline{\mathbb{L}}$.

\section{§2. Multidimensional Residue}

We recall the definition and some fundamental properties of multidimensional residue theory. 


\section{§2.1. Definitions and properties}

Let $F_{1}, \ldots, F_{n}$ be elements in $\mathbb{K}[x]$ which generate an ideal $I$ and define a non-empty and finite algebraic subvariety $\mathcal{Z}=\mathcal{Z}\left(F_{1}, \ldots, F_{n}\right)$ in $\mathbb{C}^{n}$. For any $\alpha \in \mathcal{Z}$ and $H \in \mathbb{K}[x]$, the local residue $\operatorname{res}_{F, \alpha}(H)$ of $H$ in $\alpha$ with respect to the $\operatorname{map} F=\left(F_{1}, \ldots, F_{n}\right): \mathbb{K}^{n} \rightarrow \mathbb{K}^{n}$ is

$$
\operatorname{res}_{F, \alpha}(H):=\frac{1}{(2 i \pi)^{n}} \int_{\partial V_{\alpha}} \frac{H(x) \sum_{j=1}^{n}(-1)^{j-1} \overline{F_{j}(x)} \overline{d F_{[j]}} \wedge d x}{\left(\left|F_{1}(x)\right|^{2}+\cdots+\left|F_{n}(x)\right|^{2}\right)^{n}}
$$

where $V_{\alpha}$ is an open neighborhood of $\alpha$ in $\mathbb{C}^{n}$ with a smooth real manifold boundary of dimension $2 n-1$ such that $\alpha$ is the only point in $\mathcal{Z}$ lying in $\overline{V_{\alpha}}$, $d x=d x_{1} \wedge \ldots \wedge d x_{n}$ and $d F_{[j]}=d F_{1} \wedge \cdots \wedge d F_{j-1} \wedge d F_{j+1} \wedge \cdots \wedge d F_{n}$. The sign ${ }^{-}$denotes the complex conjugate of a complex number or the conjugate of a complex function, and $\left|F_{i}(x)\right|$ the modulus of the complex number $F_{i}(x)$.

The (global) residue of $H$ with respect to $F$ is

$$
\operatorname{Res}_{F}(H):=\sum_{\alpha \in \mathcal{Z}} \operatorname{res}_{F, \alpha}(H) .
$$

We may also define the residue in an algebraic way (see [28], [26]). Let us now recall some important properties of this tool (for details see [1], [26], [3]) :

- if $H$ is in the ideal $I$ of $\mathbb{K}[x]$ generated by $F_{1}, \ldots, F_{n}$, then $\operatorname{Res}_{F}(H)=0$;

- if $\mu_{\alpha}$ denotes the multiplicity of the root $\alpha \in \mathcal{Z}$, then

$$
\operatorname{Res}_{F}(H \operatorname{Jac}(F))=\sum_{\alpha \in \mathcal{Z}} \mu_{\alpha} H(\alpha)
$$

in particular $\operatorname{Res}_{F}(\operatorname{Jac}(F))=\operatorname{dim}_{\mathbb{K}}(\mathbb{K}[x] / I)$;

- if all the points of $\mathcal{Z}$ are simple (in that case, the Jacobian of $F$ does not vanish on the variety $\mathcal{Z}$ ), then

$$
\operatorname{Res}_{F}(H)=\sum_{\alpha \in \mathcal{Z}} \frac{H(\alpha)}{\operatorname{Jac}(F)(\alpha)}
$$

- (continuity principle) : let $\epsilon \in \mathbb{C}^{n}$ be a vector of parameters and $F_{\epsilon}=$ $\left(F_{\epsilon, 1}, \ldots, F_{\epsilon, n}\right)$ be a polynomial perturbation of $F$ such that $F_{0}=F$; in a small neighborhood $V_{\alpha}$ of a root $\alpha$ of $F$, the polynomials $F_{\epsilon, 1}, \ldots, F_{\epsilon, n}$ have (for generic small values of $\epsilon$ ) $\mu_{\alpha}$ simple roots, and we have

$$
\operatorname{res}_{F, \alpha}(H)=\lim _{\epsilon \rightarrow 0} \sum_{\alpha_{\epsilon} \in \mathcal{Z}\left(F_{\epsilon, 1}, \ldots, F_{\epsilon, n}\right) \cap V_{\alpha}} \operatorname{res}_{F_{\epsilon}, \alpha_{\epsilon}}(H)
$$


- in the univariate case,

(2.2) $\operatorname{Res}_{F}(H)=$ constant coefficient of the Laurent series of $\frac{x H(x)}{F(x)}$.

- in the multivariate case :

- if for each $i=1, \ldots, n, F_{i}$ depends only on the variable $x_{i}$, then

$$
\operatorname{Res}_{F}\left(x_{1}^{m_{1}} \ldots x_{n}^{m_{n}}\right)=\operatorname{Res}_{F_{1}}\left(x_{1}^{m_{1}}\right) \ldots \operatorname{Res}_{F_{n}}\left(x_{n}^{m_{n}}\right) .
$$

- if for each $i=1, \ldots, n, F_{i}(x)=\lambda_{i} x_{i}^{D_{i}}+Q_{i}(x)$ with $\lambda_{i} \in \mathbb{K}^{*}$ and $\operatorname{deg} Q_{i}<$ $D_{i}$, then for $H \in \mathbb{K}[x], \operatorname{Res}_{F}(H)$ is the constant coefficient in the Laurent following series (in fact Laurent polynomial)

$$
\frac{H(x)}{\prod_{i=1}^{n} \lambda_{i} x_{i}^{D_{i}-1}} \sum_{\left\{m \in \mathbb{N}^{n}:|m| \leq \operatorname{deg} H+n-D_{1}-\cdots-D_{n}\right\}}(-1)^{|m|} \frac{\prod_{i=1}^{n} Q_{i}^{m_{i}}(x)}{\left(\prod_{i=1}^{n} \lambda_{i} x_{i}^{D_{i}}\right)^{m_{i}}}
$$

The computation of multidimensional residues is much more difficult in general than in the particular situations quoted above. However, we will show in the following how to compute effectively $\operatorname{Res}_{F}(H)$ for an arbitrary polynomial map $F=\left(F_{1}, \ldots, F_{n}\right)$ defining a non-empty and finite algebraic subvariety in $\mathbb{C}^{n}$.

A powerful tool in multidimensional residue theory, that we will precisely need to perform such computation, is the following generalization of the classical Transformation Law (see [6]).

Proposition 2.1. (Generalized Transformation Law) Let $\left(F_{0}, \ldots, F_{n}\right)$ and $\left(G_{0}, \ldots, G_{n}\right)$ be two maps of $\mathbb{K}\left[x_{0}, x\right]:=\mathbb{K}\left[x_{0}, \ldots, x_{n}\right]$ which define finite affine varieties in $\mathbb{C}^{n+1}$; we assume that $F_{0}=G_{0}$ and there exist an $n$-tuple of integers $m=\left(m_{1}, \ldots, m_{n}\right) \in \mathbb{N}^{n}$ and polynomials $A_{i, j} \in \mathbb{K}\left[x_{0}, x\right], i, j=$ $1, \ldots, n$, such that

$$
\forall i=1, \ldots, n, \quad F_{0}^{m_{i}} G_{i}=\sum_{j=1}^{n} A_{i, j} F_{j} ;
$$

then one has for every $H \in \mathbb{K}\left[x_{0}, x\right]$,

$$
\operatorname{Res}_{\left(F_{0}, \ldots, F_{n}\right)}(H)=\operatorname{Res}_{\left(G_{0}^{|m|+1}, G_{1} \ldots, G_{n}\right)}\left(H \operatorname{det}\left(A_{i, j}\right)\right) .
$$

If $F_{0}=x_{0}$ and $m_{1}=\cdots=m_{n}=0$, this Generalized Transformation Law reduces to the classical one : 
Corollary 2.1 (Transformation Law [28], [1]). Let $F=\left(F_{1}, \ldots, F_{n}\right)$ and $G=\left(G_{1}, \ldots, G_{n}\right)$ be two polynomial maps in $\mathbb{K}[x]$ such that the subvariety $\mathcal{Z}\left(F_{1}, \ldots, F_{n}\right)$ and $\mathcal{Z}\left(G_{1}, \ldots, G_{n}\right)$ are finite, and there are elements $A_{i, j} \in \mathbb{K}[x]$ which satisfy

$$
\forall i=1, \ldots, n, \quad G_{i}=\sum_{j=1}^{n} A_{i, j} F_{j} ;
$$

then one has for all $H \in \mathbb{K}[x], \operatorname{Res}_{F}(H)=\operatorname{Res}_{G}\left(H \operatorname{det}\left(A_{i, j}\right)\right)$.

\section{§2.2. Residue calculus}

When $F=\left(F_{1}, \ldots, F_{n}\right)$ is proper as a polynomial map from $\mathbb{C}^{n}$ to $\mathbb{C}^{n}$ (this means that for each $i \in\{1, \ldots, n\}$ there exist non-negative integer $\delta_{i}$ and polynomials $a_{i j} \in \mathbb{K}\left[u_{1}, \ldots, u_{n}\right]$ such that

$$
\left.x_{i}^{\delta_{i}}+a_{i 1}\left(F_{1}, \ldots, F_{n}\right) x_{i}^{\delta_{i}-1}+\cdots+a_{i \delta_{i}}\left(F_{1}, \ldots, F_{n}\right) \equiv 0\right),
$$

the computation of multidimensional residue with respect to $F$ is a simple consequence of these algebraic relations relating $x_{i}, F_{1}, \ldots, F_{n}$, and the Transformation Law. We now present a new algorithm which computes effectively $\operatorname{Res}_{F}$ without the properness assumption on the map $F$.

Let $D_{i}=\operatorname{deg} F_{i}, i=1, \ldots, n$, we introduce $u=\left(u_{1}, \ldots, u_{n}\right) \in \mathbb{C}^{n}$ and $\lambda \in$ $\mathbb{C}$ that we view as parameters; we consider the auxiliary system of polynomials $\Phi(\lambda, u ; x)=\left(\Phi_{1}(\lambda, u ; x), \ldots, \Phi_{n}(\lambda, u ; x)\right)$ with

$$
\Phi_{j}(\lambda, u ; x):=\lambda x_{j}^{D_{j}+1}+F_{j}(x)-u_{j} \text { for } j=1, \ldots, n .
$$

We notice that for a polynomial $H$, thanks to formula (2.4), on get a rational function $\operatorname{Res}_{\Phi(\lambda, u ; .)}(H) \in \mathbb{K}\left[\frac{1}{\lambda}, u_{1}, \ldots, u_{n}\right]$. A strategy to determine the scalar $\operatorname{Res}_{F}(H)$ could be to follow this rational function when $(\lambda, u)$ tends to $(0,0)$. But this is not straightforward as shown by the following simple example : $n=2, F_{1}=x_{1}, F_{2}=x_{2}, \operatorname{Res}_{F}(1)=1$, whereas $\operatorname{Res}_{\Phi(\lambda, u ; .)}(1) \equiv 0$. The problem comes from the fact that the perturbation $\Phi(\lambda, u ;$.$) introduces new zeroes which$ disappear to infinity when the parameters $(\lambda, u)$ tend to $(0,0)$. In other words, the residue "blows-up".

By Bézout's theorem the number of points in the subvariety $\mathcal{Z}_{\lambda, u}$ in $\mathbb{C}^{n}$ defined by $\Phi_{1}(\lambda, u ; x), \ldots, \Phi_{n}(\lambda, u ; x)$ is $N=\left(D_{1}+1\right) .\left(D_{2}+1\right) \ldots\left(D_{n}+1\right)$.

Fix $i \in\{1, \ldots, n\}$. Denote by $\alpha_{1}^{(i)}(\lambda, u), \ldots, \alpha_{N}^{(i)}(\lambda, u)$ the $i^{t h}$ coordinates of the $N$ points of $\mathcal{Z}_{\lambda, u}$. Using formulae (2.1) and (2.4), we observe that for 
$k \in\{1, \ldots, N\}$, the Newton sum

$$
S_{k}^{(i)}(\lambda, u)=\sum_{j=1}^{N}\left(\alpha_{j}^{(i)}(\lambda, u)\right)^{k} \in \mathbb{K}(\lambda)[u],
$$

appears as the constant coefficient of the Laurent polynomial

$$
\frac{x_{i}^{k} \operatorname{Jac}(\Phi(\lambda, u ; x))}{\lambda^{n} x_{1}^{D_{1}} \ldots x_{n}^{D_{n}}} \sum_{m \in \mathbb{N}^{n}:|m| \leq k}(-1)^{|m|} \prod_{i=1}^{n}\left(\frac{F_{i}(x)-u_{i}}{\lambda x_{i}^{D_{i}+1}}\right)^{m_{i}} .
$$

By means of the Newton formulae, for $k=1, \ldots, N$,

$$
k \sigma_{k}^{(i)}=-S_{k}^{(i)}-S_{k-1}^{(i)} \sigma_{1}^{(i)}-\cdots-S_{1}^{(i)} \sigma_{k-1}^{(i)},
$$

one can compute easily, from the $S_{k}^{(i)}(\lambda, u), k=1, \ldots, N$, the elementary symmetric functions $\sigma_{k}^{(i)}(\lambda, u), k=1, \ldots, N$, of the set $\left\{\alpha_{1}^{(i)}(\lambda, u), \ldots, \alpha_{N}^{(i)}(\lambda, u)\right\}$. Therefore one get explicitly the univariate polynomial in $x_{i}$ :

$$
\begin{aligned}
\prod_{k=1}^{N}\left(x_{i}-\alpha_{k}^{(i)}(\lambda, u)\right) & =x_{i}^{N}+\sigma_{1}^{(i)}(\lambda, u) x_{i}^{N-1}+\cdots+\sigma_{N}^{(i)}(\lambda, u) \\
& =\frac{\mathcal{N}_{i}\left(\lambda, u, x_{i}\right)}{\mathcal{D}_{i}(\lambda)}
\end{aligned}
$$

where $\mathcal{N}_{i} \in \mathbb{K}\left[\lambda, u, x_{i}\right]$ and $\mathcal{D}_{i}(\lambda) \in \mathbb{K}[\lambda]$ are relatively prime. By definition of the algebraic variety $\mathcal{Z}_{\lambda, u}$, the polynomial $\mathcal{N}_{i}$ satisfies the algebraic relation

$$
\mathcal{N}_{i}\left(\lambda, \lambda x_{1}^{D_{1}+1}+F_{1}(x), \ldots, \lambda x_{n}^{D_{n}+1}+F_{n}(x), x_{i}\right) \equiv 0
$$

in $\mathbb{K}\left[\lambda, x_{1}, \ldots, x_{n}\right]$. As $u_{i}=x_{i}^{D_{i}+1}+F_{i}-\Phi_{i}$, for $i=1, \ldots, n$, we deduce from (2.8) the existence of polynomials $A_{i, j}$ such that

$$
\mathcal{N}_{i}\left(\lambda, u_{1}, \ldots, u_{n}, x_{i}\right)=\sum_{j=1}^{n} \Phi_{j}(\lambda, u ; x) A_{i, j}(\lambda, u, x) .
$$

We choose a vector $\alpha=\left(\alpha_{0}, \ldots, \alpha_{n}\right) \in \mathbb{K}^{n+1}$ and we set $\lambda=\alpha_{0} x_{0}, u_{1}=$ $\alpha_{1} x_{0}, \ldots, u_{n}=\alpha_{n} x_{0}$. This amounts to specify a controlled line in the space of parameters $(\lambda, u)$.

Let $m_{i}$ be the valuation of $\mathcal{N}_{i}\left(\lambda, u, x_{i}\right)$ as a polynomial in $\lambda$ and $u$. Then there exist two unique polynomials $R_{i} \in \mathbb{K}\left[x_{i}\right]$ and $S_{i} \in \mathbb{K}\left[x_{0}, x_{i}\right]$ such that

$$
\mathcal{N}_{i}\left(\alpha x_{0}, x_{i}\right)=x_{0}^{m_{i}}\left(R_{i}\left(\alpha ; x_{i}\right)-x_{0} S_{i}\left(\alpha ; x_{0}, x_{i}\right)\right) .
$$

So to each map $F=\left(F_{1}, \ldots, F_{n}\right)$, one can associate three families of polynomials $R_{i}\left(\alpha ; x_{i}\right), S_{i}\left(\alpha ; x_{0}, x_{i}\right), i=1, \ldots, n$, and $A_{i, j}(\lambda, u, x), i, j=1, \ldots, n$. 
Theorem 2.1. Let $F=\left(F_{1}, \ldots, F_{n}\right)$ be a polynomial map such that the algebraic affine variety $\mathcal{Z}\left(F_{1}, \ldots, F_{n}\right)$ is non-empty and finite. We assume that for $\alpha \in \mathbb{K}^{n+1}$, the polynomial $R_{1}\left(\alpha ; x_{1}\right) \ldots R_{n}\left(\alpha ; x_{n}\right) \neq 0$. For each $k \in \mathbb{N}^{n}$, we set $S^{k}=S_{1}^{k_{1}} \cdot S_{2}^{k_{2}} \ldots S_{n}^{k_{n}}$, and $R^{k+1}=\left(R_{1}^{k_{1}+1}, \ldots, R_{n}^{k_{n}+1}\right)$. Then for every $H \in \mathbb{K}[x]$, we have

$$
\operatorname{Res}_{F}(H)=\sum_{k \in \mathbb{N}^{n}:|k| \leq|m|} \operatorname{Res}_{\left(x_{0}^{|m|-|k|+1}, R^{k+1}\right)}\left(H S^{k} \operatorname{det}\left(B_{i j}\right)\right),
$$

with $B_{i j}=A_{i, j}\left(\alpha x_{0}, x\right) \in \mathbb{K}\left[x_{0}, x\right], i, j=1, \ldots, n$.

Proof. If $G=\left(x_{0}, F_{1}+x_{0}\left(\alpha_{0} x_{1}^{D_{1}+1}-\alpha_{1}\right), \ldots, F_{n}+x_{0}\left(\alpha_{0} x_{n}^{D_{n}+1}-\alpha_{n}\right)\right)$, and $\tilde{F}=\left(x_{0}, F_{1}, \ldots, F_{n}\right)$, we have $G=T \tilde{F}$ with

$$
T=\left(\begin{array}{ccccc}
1 & 0 & \ldots & 0 \\
\alpha_{0} x_{1}^{D_{1}+1}-\alpha_{1} & 1 & 0 & \ldots & 0 \\
\vdots & \ddots & \ddots & \vdots \\
\alpha_{0} x_{n}^{D_{n}+1}-\alpha_{n} & 0 & \ldots & \ldots
\end{array}\right)
$$

As $\operatorname{det}(T)=1$, it follows from the Transformation Law that for $H \in \mathbb{K}[x]$, $\operatorname{Res}_{\tilde{F}}(H)=\operatorname{Res}_{G}(H)$.

Now, if the points of the subvariety $\mathcal{Z}\left(F_{1}, \ldots, F_{n}\right)$ are simple, it is easy to check that $\operatorname{Res}_{\tilde{F}}(H)=\operatorname{Res}_{F}(H)$; moreover the continuity principle implies that $\operatorname{Res}_{\tilde{F}}(H)=\operatorname{Res}_{F}(H)$ even if some of the common roots of $F_{1}, \ldots, F_{n}$ are not simple. Consequently $\operatorname{Res}_{F}(H)=\operatorname{Res}_{G}(H)$.

As a consequence of the Generalized Transformation Law, formulae (2.9) and (2.10) and $\Phi_{j}\left(\alpha x_{0} ; x\right)=F_{j}+x_{0}\left(\alpha_{0} x_{j}^{D_{j}+1}-\alpha_{j}\right)$, we have

$$
\operatorname{Res}_{G}(H)=\operatorname{Res}_{\left(x_{0}^{|m|+1}, R-x_{0} S\right)}\left(H \operatorname{det}\left(B_{i, j}\right)\right),
$$

where $R-x_{0} S=\left(R_{1}-x_{0} S_{1}, \ldots, R_{n}-x_{0} S_{n}\right)$. Using the following trivial identities, for $i=1, \ldots, n$,

$$
R_{i}^{|m|+1}=\left(x_{0} S_{i}\right)^{|m|+1}+\left(R_{i}-x_{0} S_{i}\right) \sum_{k_{i}=0}^{|m|}\left(x_{0} S_{i}\right)^{k_{i}} R_{i}^{|m|-k_{i}},
$$

the Transformation Law applied to $\left(x_{0}^{|m|+1}, R-x_{0} S\right)$ and $\left(x_{0}^{|m|+1}, R^{|m|+1}\right)$, we get

$$
\begin{aligned}
\operatorname{Res}_{F}(H) & =\operatorname{Res}_{\left(x_{0}^{|m|+1}, R^{|m|+1}\right)}\left(H \operatorname{det}\left(B_{i j}\right) \sum_{k} x_{0}^{|k|} S^{k} R_{1}^{|m|-k_{1}} \ldots R_{n}^{|m|-k_{n}}\right) \\
& =\sum_{|k| \leq|m|} \operatorname{Res}_{\left(x_{0}^{|m|-|k|+1}, R^{k+1}\right)}\left(H S^{k} \operatorname{det}\left(B_{i j}\right)\right)
\end{aligned}
$$


Note that the expression of $\operatorname{Res}_{F}(H)$ given in the previous result can be computed using formulae (2.2) and (2.3). Thus the computation of the multidimensional residue is reduced to the univariate case via this theorem.

Remark 2.1. We recall that a sequence of polynomials $F_{1}, \ldots, F_{n}$ in $\mathbb{K}[x]$ is quasi-regular if the Koszul complex over $\mathbb{K}[x]$ associated to this sequence is exact except possibly in degree 0 . If $I$ is the ideal generated by $F_{1}, \ldots, F_{n}$, this is equivalent to the following : for every $d \in \mathbb{N}$, and $a_{k} \in \mathbb{K}[x]$, if

$$
\sum_{k=\left(k_{1}, \ldots, k_{n}\right) \in \mathbb{N}^{n}:|k|=d} a_{k} P_{1}^{k_{1}} \ldots P_{n}^{k_{n}} \in I^{d+1},
$$

then the elements $a_{k}$ are in $I$ (see [27]).

We can write for $i=1, \ldots, n$, the identities (2.8) as

$$
\sum_{\delta=\left(\delta_{0}, \ldots, \delta_{n}\right)} \Delta_{i, \delta}\left(x_{i}\right) \lambda^{\delta_{0}}\left(\lambda x_{1}^{D_{1}+1}+F_{1}(x)\right)^{\delta_{1}} \ldots\left(\lambda x_{n}^{D_{n}+1}+F_{n}(x)\right)^{\delta_{n}}=0 .
$$

Since the map $\left(\lambda, \lambda x_{1}^{D_{1}+1}+F_{1}(x), \ldots, \lambda x_{n}^{D_{n}+1}+F_{n}(x)\right)$ is quasi-regular (because this sequence define a finite subvariety in $\left.\mathbb{C}^{n+1}\right)$, the univariate polynomials $\Delta_{i, \delta}\left(x_{i}\right)$ with the smallest $|\delta|=\delta_{0}+\cdots+\delta_{n}$ are contained in the ideal of $\mathbb{K}\left[\lambda, x_{1}, \ldots, x_{n}\right]$ generated by $\lambda, \lambda x_{1}^{D_{1}+1}+F_{1}(x), \ldots, \lambda x_{n}^{D_{n}+1}+F_{n}(x)$. So these univariate polynomials vanish on the common roots of $F_{1}, \ldots, F_{n}$. In this way we can determine the algebraic affine variety $\mathcal{Z}\left(F_{1}, \ldots, F_{n}\right)$ by solving these univariate polynomials.

We recall that if a polynomial $F=\sum_{\alpha} c_{\alpha} x^{\alpha} \in \mathbb{Z}\left[x_{1}, \ldots, x_{n}\right]$, the (naive) height of $F$ is $h(F)=\max _{\alpha}\left(\log \left|c_{\alpha}\right|\right)$, and that the height of $\frac{a}{b} \in \mathbb{Q}$ with $\operatorname{gcd}(a, b)=1$ is $\max (\log |a|, \log |b|)$. Now if the coefficients of $F_{1}, \ldots, F_{n}, H$ are integers of maximal height $h$, using the previous method of computation of residue we can express $\operatorname{Res}_{F}(H)$ as a rational function of height bounded essentially by $D^{n} h$, where $D=\max \left(\operatorname{deg} F_{1}, \ldots, \operatorname{deg} F_{n}, \operatorname{deg} H\right)$.

Remark 2.2. In order to reduce the size of integers $N$ (used to construct the polynomial $\mathcal{N}_{i}\left(\lambda, u, x_{i}\right)$ in (2.7)) and $|m|$ in Theorem 2.1, we can perturb the original polynomial map $F$ in a more subtle way using a generalization of the formula (2.4). It was already noticed in [1] that, if for each $i=1, \ldots, n, \Phi_{i}(x)=$ $\lambda_{i} x_{i}^{D_{i}}+\sum_{j=1}^{i-1} x_{j} \Phi_{i j}(x)+Q_{i}(x)$ with $\lambda_{i} \in \mathbb{K}^{*}, \Phi_{i j}$ is a homogeneous polynomial of degree $D_{i}-1$ and $\operatorname{deg} Q_{i}<D_{i}$, then for any $H \in \mathbb{K}[x], \operatorname{Res}_{\Phi}(H)$ is the constant coefficient in the Laurent polynomial

$$
\frac{H(x)}{\prod_{i=1}^{n} \lambda_{i} x_{i}^{D_{i}-1}} \sum_{m}(-1)^{|m|} \frac{\prod_{i=1}^{n}\left(\Phi_{i}(x)-\lambda_{i} x_{i}^{D_{i}}\right)^{m_{i}}}{\left(\prod_{i=1}^{n} \lambda_{i} x_{i}^{D_{i}}\right)^{m_{i}}} .
$$


This remark will be used in Examples 1, 2 and 3.

Theorem 2.1 gives rise to the following algorithm :

Algorithm 2.1 (computation of the residue of a polynomial map). INPUTS : a map $F=\left(F_{1}, \ldots, F_{n}\right)$ in $\mathbb{K}[x]$ such that the affine subvariety $\mathcal{Z}\left(F_{1}, \ldots, F_{n}\right)$ is non-empty and finite; a polynomial $H \in \mathbb{K}[x]$.

Step 1. let $\lambda \in \mathbb{C}$ and $u=\left(u_{1}, \ldots, u_{n}\right) \in \mathbb{C}^{n}$ be parameters; set

$$
\Phi(\lambda, u ; x)=\left(\Phi_{1}(\lambda, u ; x), \ldots, \Phi_{n}(\lambda, u ; x)\right)
$$

where $\Phi_{i}(\lambda, u ; x)=\lambda x_{i}^{\operatorname{deg} F_{i}+1}+F_{i}(x)-u_{i}, i=1, \ldots, n$, (taking into account Remark 2.2, if there exist a homogeneous polynomial $F_{i j}$ of degree $\operatorname{deg} F_{i}-1$ and a polynomial $Q_{i}$ of degree at most $\operatorname{deg} F_{i}-1$ such that $F_{i}=\sum_{j=1}^{i-1} x_{j} F_{i j}+Q_{i}$, we set $\left.\Phi_{i}(\lambda, u ; x)=\lambda x_{i}^{\operatorname{deg} F_{i}}+F_{i}(x)-u_{i}\right)$; determine for each $i=1, \ldots, n$, and for each $j=1, \ldots, N=\operatorname{deg} \Phi_{1} \cdots \operatorname{deg} \Phi_{n}$, the constant coefficient $S_{j}^{(i)}(\lambda, u)$ in the Laurent polynomial given by the formula (2.5) (or (2.11)).

Step 2. use Newton formulae (2.6) to compute the $j^{\text {th }}$ elementary symmetric functions $\sigma_{j}^{(i)}(\lambda, u) \in \mathbb{K}(\lambda)[u], j=1, \ldots, N, i=1, \ldots, n$, and to obtain the univariate polynomials $\mathcal{N}_{i}\left(\lambda, u, x_{i}\right) \in \mathbb{K}\left[\lambda, u, x_{i}\right], i=1, \ldots, n$, given by $(2.7)$.

Step 3. choose a random $\alpha=\left(\alpha_{0}, \ldots, \alpha_{n}\right) \in \mathbb{C}^{n+1}$; express each polynomial $\mathcal{N}_{i}\left(\alpha x_{0}, x_{i}\right)$ as

$$
\begin{aligned}
\mathcal{N}_{i}\left(\alpha x_{0}, x_{i}\right) & =x_{0}^{m_{i}}\left(R_{i}\left(\alpha ; x_{i}\right)-x_{0} S_{i}\left(\alpha ; x_{0}, x_{i}\right)\right) \\
& =\sum_{j=1}^{n} \Phi_{j}\left(\alpha x_{0} ; x\right) A_{i, j}\left(\alpha x_{0}, x\right) .
\end{aligned}
$$

Check that $R_{1}\left(\alpha ; x_{1}\right) \ldots R_{n}\left(\alpha ; x_{n}\right) \neq 0$, if not try another choice of $\alpha$.

Step 4. use formulae (2.3) and (2.2) to compute the expression

$$
\sum_{|k| \leq|m|} \operatorname{Res}_{\left(x_{0}^{|m|-|k|+1}, R^{k+1}\right)}\left(H S^{k} \operatorname{det}\left(A_{i j}\left(\alpha x_{0}, x\right)\right)\right)
$$

of $\operatorname{Res}_{\mathrm{F}}(\mathrm{H})$ given by Theorem 2.1 .

OUtPut : $\operatorname{Res}_{F}(H) \in \mathbb{K}$.

Let $F=\left(F_{1}, \ldots, F_{n}\right)$ be a polynomial map such that the affine variety $\mathcal{Z}\left(F_{1}, \ldots, F_{n}\right)$ is finite. For every $i=1, \ldots, n$, there exist polynomials $a_{i j}$ in $\mathbb{K}\left[u_{1}, \ldots, u_{n}\right]$ and a positive integer $\delta_{i}$ such that

$$
P_{i}\left(u_{0}, \ldots, u_{n}\right)=\sum_{j=0}^{\delta_{i}} a_{i j}\left(u_{1}, \ldots, u_{n}\right) u_{0}^{\delta_{i}-j}
$$


satisfies the algebraic identity $P_{i}\left(x_{i}, F_{1}, \ldots, F_{n}\right) \equiv 0$. When the $n$ elements $a_{10}, \ldots, a_{n 0}$ are non-vanishing constants, the map $F$ is proper.

Now if for each $i=1, \ldots, n$, there exists $j$ in $\left\{0, \ldots, \delta_{i}-1\right\}$ with $a_{i j}(0) \neq 0$, then we deduce from this identity that an univariate non-constant polynomial in $x_{i}$ belongs to the ideal generated by $F_{1}, \ldots, F_{n}$. In this case, we can compute the residue with respect to $F$ using only the Transformation Law. But if there exists $i_{0} \in\{1, \ldots, n\}$ with $a_{i j}(0)=0$ for every $j=0, \ldots, \delta_{i}-1$ as in the following example, this strategy for the computation of $\operatorname{Res}_{F}$ fails.

Example 1. We consider in $\mathbb{K}\left[x_{1}, x_{2}, x_{3}\right]$ the elements

$$
f_{1}=x_{1}, \quad f_{2}=x_{2}+x_{1} x_{2}^{2} x_{3}, \quad f_{3}=x_{3}+x_{1} x_{2} x_{3}^{2} .
$$

The irreducible algebraic relations between $f_{1}, f_{2}, f_{3}$ and the coordinate functions $x_{1}, x_{2}, x_{3}$ are

$$
P_{1}=u_{0}-u_{1}, P_{2}=u_{1} u_{3} u_{0}^{3}+u_{2} u_{0}-u_{2}^{2}, P_{3}=u_{1} u_{2} u_{0}^{3}+u_{3} u_{0} .
$$

Our algorithm can be viewed as an extension of the previous method to compute the residue with respect to any polynomial map (which defines a finite affine variety). The key point is the use of the Generalized Transformation Law.

For shortness we will illustrate the Algorithm 2.1 on a simple example for which the computation of residue can be done directly. We consider in $\mathbb{K}\left[x_{1}, x_{2}\right], F=\left(F_{1}, F_{2}\right)=\left(x_{1}, x_{1} x_{2}^{2}+x_{2}\right)$. The map $F$ is not proper because the irreducible algebraic relation between $x_{2}, F_{1}, F_{2}$ is $u_{0}^{2} u_{1}+u_{0}-u_{2}$.

Using Remark 2.2, $\Phi_{1}=\lambda x_{1}-u_{1}$, and $\Phi_{2}=\lambda x_{2}^{3}+x_{1} x_{2}^{2}+x_{2}-u_{2}$. We have $N=3, S_{1}^{(1)}=\frac{3}{\lambda} u_{1}, S_{2}^{(1)}=\frac{3}{\lambda^{2}} u_{1}^{2}, S_{3}^{(1)}=\frac{3}{\lambda^{3}} u_{1}^{3}, S_{1}^{(2)}=-\frac{1}{\lambda^{2}} u_{1}$, $S_{2}^{(2)}=-\frac{2}{\lambda}+\frac{1}{\lambda^{4}} u_{1}^{2}, S_{3}^{(2)}=\frac{3}{\lambda} u_{2}+\frac{3}{\lambda^{3}} u_{1}-\frac{1}{\lambda^{6}} u_{1}^{3}$. Then we obtain

$$
\begin{aligned}
& \mathcal{N}_{1}\left(\lambda, u_{1}, u_{2}, x_{1}\right)=\lambda x_{1}-u_{1}=\Phi_{1}, \\
& \mathcal{N}_{2}\left(\lambda, u_{1}, u_{2}, x_{2}\right)=\lambda^{2} x_{2}^{3}+x_{2}^{2} u_{1}+\lambda x_{2}-\lambda u_{2}=-x_{2}^{2} \Phi_{1}+\lambda \Phi_{2},
\end{aligned}
$$

and $\operatorname{det}\left(A_{i, j}\right)=\lambda$. We deduce that for a generic vector $\left(\alpha_{0}, \alpha_{1}, \alpha_{2}\right) \in \mathbb{K}^{3}$,

$$
\begin{aligned}
& \mathcal{N}_{1}\left(\alpha_{0} x_{0}, \alpha_{1} x_{0}, \alpha_{2} x_{0}, x_{1}\right)=x_{0}\left(\alpha_{0} x_{1}-\alpha_{1}\right), \\
& \mathcal{N}_{2}\left(\alpha_{0} x_{0}, \alpha_{1} x_{0}, \alpha_{2} x_{0}, x_{2}\right)=x_{0}\left(\left(\alpha_{0}^{2} x_{2}^{3}-\alpha_{0} \alpha_{2}\right) x_{0}+\alpha_{1} x_{2}^{2}+\alpha_{0} x_{2}\right) .
\end{aligned}
$$

So the polynomials $R_{1}=\alpha_{0} x_{1}-\alpha_{1}, R_{2}=\alpha_{1} x_{2}^{2}+\alpha_{0} x_{2}, S_{1}=0, S_{2}=-\alpha_{0}^{2} x_{2}^{3}+$ $\alpha_{0} \alpha_{2}$, and the integers $m_{1}=1, m_{2}=1$. Finally for every $H$,

$$
\operatorname{Res}_{F}(H)=\sum_{\left(k_{1}, k_{2}\right): k_{1}+k_{2} \leq 2} \operatorname{Res}_{\left(x_{0}^{3-k_{1}-k_{2}}, R_{1}^{k_{1}+1}, R_{2}^{k_{2}+1}\right)}\left(\alpha_{0} x_{0} H S_{1}^{k_{1}} S_{2}^{k_{2}}\right) .
$$


Remark 2.3. Computations in Step 3 and Step 4 of the previous algorithm may be performed for a random $\alpha$ even if nothing is assumed with respect to the geometry of the zero set $\mathcal{Z}\left(F_{1}, \ldots, F_{n}\right)$. If two distinct generic values of $\alpha \in \mathbb{C}^{n+1}$ lead to distinct results in Step 4 , then $\mathcal{Z}\left(F_{1}, \ldots, F_{n}\right)$ is non-finite ; so the residue calculus described in this subsection allows also to decide whether $F_{1}, \ldots, F_{n}$ define a finite subvariety in $\mathbb{C}^{n}$ or not.

\section{§3. Applications in CAGD}

The residue calculus developed above can be used in order to study some important problems in CAGD. In this field, hypersurfaces (essentially curves and surfaces) are often given parametrically. But some basic operations, such as the determination of the singular locus and the intersection problem are better treated via an implicit equation.

\section{§3.1. Implicitization problem}

A rational hypersurface $(H)$ in the affine space $\mathbb{K}^{n+1}$ can be represented by a parametric representation

$$
\left\{\begin{array}{c}
x_{0}=\frac{f_{0}\left(s_{1}, \ldots, s_{n}\right)}{d_{0}\left(s_{1}, \ldots, s_{n}\right)} \\
\vdots \\
x_{n}=\frac{f_{n}\left(s_{1}, \ldots, s_{n}\right)}{d_{n}\left(s_{1}, \ldots, s_{n}\right)}
\end{array}\right.
$$

where $f_{0}, \ldots, f_{n}, d_{0}, \ldots, d_{n}$ are polynomials in the variables $s_{1}, \ldots, s_{n}$, with coefficients in the subfield $\mathbb{K}$ of $\mathbb{C}$, or by an implicit equation (i.e. an element $Q$ in $\mathbb{K}\left[x_{0}, \ldots, x_{n}\right]$ of minimal degree satisfying $Q(\alpha)=0$ for all $\alpha$ in $\left.(H)\right)$. These two representations are important for different reasons. For instance, the first one is useful to draw $(H)$ and the second one to decide whether a point is in the given hypersurface or not.

Here we are interested to the problem of converting a parametric representation into an implicit one. Classically the solution of this question is given by elimination theory through essentially two classes of tools : resultants and Gröbner bases. On one side, techniques based on resultants (given by matrices) may produce extraneous terms along with the implicit equation ; and expanding such an implicit equation (or a multiple of it) in the monomial basis is time consuming. Moreover resultants identically vanish when base-points (that is, common roots of $f_{0}, \ldots, f_{n}, d_{0}, \ldots, d_{n}$ ) are present. On the other side, methods 
based on Gröbner bases computations are fairly expensive in practice and also unstable.

Other techniques exist (for example, based on residual resultants [8], moving surfaces [30], [9], approximation complexes [10]). These methods are only valid under restrictive geometric hypotheses on the zero-locus of base-points (assumed to be known) which are hard to verify. In [24] the theory of multivariate residues is used to implicitize surfaces of a very special type (i.e. the equations of the surface have no common zeroes at infinity) ; such results were recently extended in [22] to general surfaces using bezoutian matrices. We pursue these last approaches for the implicitization problem of a rational hypersurface $(H)$ thanks to the presented algorithm in subsection 2.2 for the computation of the multidimensional residue. Our method works in the presence of base-points and no geometric assumption on the zero-locus of base-points is needed ; compared to the method proposed in [22], its advantage lies in the fact that instead of computing huge symbolic determinants, one has to use only some specific coefficients in Laurent developments.

Let us consider the following $n+1$ polynomials in the polynomial ring $\left(\mathbb{K}\left[x_{0}, x\right]\right)[s]:=\left(\mathbb{K}\left[x_{0}, \ldots, x_{n}\right]\right)\left[s_{1}, \ldots, s_{n}\right]$ extracted from the parametrization (3.1):

$$
\left\{\begin{array}{c}
F_{0}(s)=x_{0} d_{0}(s)-f_{0}(s) \\
\vdots \\
F_{n}(s)=x_{n} d_{n}(s)-f_{n}(s) ;
\end{array}\right.
$$

let $\mathcal{Z}:=\mathcal{Z}\left(F_{1}, \ldots, F_{n}\right)=\left\{\alpha \in \overline{\mathbb{K}}(x)^{n} ; F_{1}(\alpha)=\cdots=F_{n}(\alpha)=0\right\}$, and $d:=d_{0} \ldots d_{n}$. We write $\mathcal{Z}$ as $\mathcal{Z}=Z_{1} \cup \mathcal{Z}_{2}$, where

$$
\mathcal{Z}_{1}:=\mathcal{Z} \cap \mathcal{Z}(d)=\left\{\alpha \in \overline{\mathbb{K}}(x)^{n} ; F_{1}(\alpha)=\cdots=F_{n}(\alpha)=d(\alpha)=0\right\}
$$

and $\mathcal{Z}_{2}:=\mathcal{Z} \backslash \mathcal{Z}_{1}$. Let also denote by $F$ the map $\left(F_{1}, \ldots, F_{n}\right)$. Using the identity (2.1) and the multidimensional residue calculus presented in Subsection 2.2, we can test whether $\mathcal{Z}_{2}$ is finite or not. In order to do that, let $s_{n+1}$ be a new variable and $F_{n+1}\left(s, s_{n+1}\right):=s_{n+1} d(s)-1$; if we denote by $\mathcal{W}$ the zero set defined by $F_{1}, \ldots, F_{n+1}$ in $\overline{\mathbb{K}}(x)^{n+1}$, then it is clear that

$$
\mathcal{W}=\left\{(\alpha, 1 / d(\alpha)) \in \overline{\mathbb{K}}(x)^{n+1} ; \alpha \in \mathcal{Z}_{2}\right\}
$$

Moreover the multiplicity of $\alpha$ as a common root of $F_{1}, \ldots, F_{n}$ equals the multiplicity of $(\alpha, 1 / d(\alpha))$ as an element in $\mathcal{W}$; so, provided $\mathcal{Z}_{2}$ is finite, counting multiplicities, the number of points in $\mathcal{Z}_{2}$ is equal to

$$
\sum_{\alpha \in \mathcal{Z}_{2}} \operatorname{res}_{F, \alpha}(\operatorname{Jac}(F))=\operatorname{Res}_{\left(F, F_{n+1}\right)}\left(\operatorname{Jac}\left(F, F_{n+1}\right)\right),
$$


where $\left(F, F_{n+1}\right)=\left(F_{1}, \ldots, F_{n}, F_{n+1}\right)$. This shows that the computation of $\operatorname{Res}_{\left(F, F_{n+1}\right)}\left(\operatorname{Jac}\left(F, F_{n+1}\right)\right)$ (which should be a rational function in $x$, actually a constant), conducted as in Subsection 2.2 for $x$ generic, provides a test to check whether $\mathcal{Z}_{2}$ is finite or not.

We will assume from now on that $\mathcal{Z}_{2}$ is finite, and contains $\delta$ points (counted with multiplicities).

Let $P\left(x_{0}, x\right)$ be the following nonzero element

$$
\begin{aligned}
P\left(x_{0}, x\right) & =\prod_{\alpha \in \mathcal{Z}_{2}} F_{0}(\alpha)=\left(\prod_{\alpha \in \mathcal{Z}_{2}} d_{0}(\alpha)\right)\left(\prod_{\alpha \in \mathcal{Z}_{2}}\left(x_{0}-\frac{f_{0}(\alpha)}{d_{0}(\alpha)}\right)\right) \\
& =\left(\prod_{\alpha \in \mathcal{Z}_{2}} d_{0}(\alpha)\right)\left(x_{0}^{\delta}+\sigma_{1}(x) x_{0}^{\delta-1}+\cdots+\sigma_{\delta}(x)\right)
\end{aligned}
$$

where $\sigma_{k}(x) \in \mathbb{K}(x), k=1, \ldots, \delta$, is the $k^{t h}$ elementary symmetric function of the set $\left\{f_{0}(\alpha) / d_{0}(\alpha) ; \alpha \in \mathcal{Z}_{2}\right\}$.

The following lemma allows to compute the power sums

$$
S_{k}(x)=\sum_{\alpha \in \mathcal{Z}_{2}}\left(\frac{f_{0}(\alpha)}{d_{0}(\alpha)}\right)^{k}, k=1, \ldots, \delta .
$$

Lemma 3.1. For every $k \in\{1, \ldots, \delta\}$, we have

$$
S_{k}(x)=\operatorname{Res}_{\left(F, F_{n+1}\right)}\left(\operatorname{Jac}\left(F, F_{n+1}\right)\left(s_{n+1} d_{1} \ldots d_{n} f_{0}\right)^{k}\right) .
$$

Proof. From (3.2) and the equality (2.1), it follows that

$$
\begin{aligned}
S_{k}(x) & =\sum_{(\alpha, 1 / d(\alpha)) \in \mathcal{W}}\left(\frac{1}{d(\alpha)} d_{1}(\alpha) \ldots d_{n}(\alpha) f_{0}(\alpha)\right)^{k} \\
& =\operatorname{Res}_{\left(F, F_{n+1}\right)}\left(\operatorname{Jac}\left(F, F_{n+1}\right)\left(s_{n+1} d_{1} \ldots d_{n} f_{0}\right)^{k}\right) .
\end{aligned}
$$

The coefficients $\sigma_{k}(x), k=1, \ldots, \delta$, in the polynomial $P\left(x_{0}, x\right)$ (see formula (3.3)) can be computed from Newton sums $S_{k}(x) \in \mathbb{K}(x)$ using Newton identities (2.6).

We recall that the squarefree part of a polynomial $Q\left(x_{0}, \ldots, x_{n}\right)$ is obtained by computing $\frac{Q}{\operatorname{gcd}\left(Q, \frac{\partial Q}{\partial x_{0}}, \ldots, \frac{\partial Q}{\partial x_{n}}\right)}$.

Theorem 3.1. Under the assumption that the affine algebraic variety $\mathcal{Z}_{2}$ is finite and contains $\delta$ (multiplicities being taken into account), an implicit 
equation of the rational hypersurface (3.1) is given by the squarefree part of the numerator of the rational function

$$
E\left(x_{0}, x\right)=x_{0}^{\delta}+\sigma_{1}(x) x_{0}^{\delta-1}+\cdots+\sigma_{\delta}(x) \in \mathbb{K}(x)\left[x_{0}\right] .
$$

Proof. The elementary symmetric functions $\sigma_{1}, \ldots, \sigma_{\delta}$ of $\left\{\frac{f_{0}(\alpha)}{d_{0}(\alpha)} ; \alpha \in \mathcal{Z}_{2}\right\}$ are elements in $\mathbb{K}(x)$ that one can express in the reduced form $\sigma_{k}=\frac{N_{k}}{D_{k}}, k=$ $1, \ldots, \delta$. Let us choose a point $x$ in the open subset $U:=\left\{x \in \mathbb{K}^{n} ; D_{1} \ldots D_{\delta}(x) \neq\right.$ $0\}$. For any $\alpha$ such that $x_{i} d_{i}(\alpha)-f_{i}(\alpha)=0, i=0, \ldots, n$, and $d(\alpha) \neq 0$, one has $E\left(x_{0}, x\right)=0$, which means that the numerator of $E$ vanishes at every point $\left(x_{0}, x\right)$ in the parametrized hypersurface $(H)$; conversely, if the numerator of $E$ vanishes at the point $\left(x_{0}, \ldots, x_{n}\right) \in \mathbb{K}^{n+1}$ with $\left(x_{1}, \ldots, x_{n}\right) \in U$, it implies that there exists $\alpha \in \mathbb{K}^{n}$ such that $x_{i} d_{i}(\alpha)-f_{i}(\alpha)=0, i=0, \ldots, n$, and $d(\alpha) \neq 0$, which means that $\left(x_{0}, x\right)$ lies in $(H)$. From this, it follows that the squarefree part of the numerator of $E$ gives (up to a scalar) the implicit equation of $(H)$.

Algorithm 3.1 (implicitization of a rational hypersurface). INPUT : an affine hypersurface in $\mathbb{K}^{n+1}$ given by (3.1).

Step 1. Set for $i=1, \ldots, n, F_{i}(s)=x_{i} d_{i}(s)-f_{i}(s) \in(\mathbb{K}[x])[s]$ and $F_{n+1}\left(s, s_{n+1}\right)=s_{n+1} d_{0}(s) \ldots d_{n}(s)-1 \in \mathbb{K}\left[s, s_{n+1}\right] ;$ let $F=\left(F_{1}, \ldots, F_{n}\right)$; through the residue computation of $\operatorname{Res}_{\left(F, F_{n+1}\right)}\left(\operatorname{Jac}\left(F, F_{n+1}\right)\right)$ performed using Algorithm 2.1, decide whether $F_{1}, \ldots, F_{n+1}$ define a finite algebraic variety.

Step 2. If the test in Step 1 is positive, for $x$ generic let

$$
\delta:=\operatorname{Res}_{\left(F, F_{n+1}\right)}\left(\operatorname{Jac}\left(F, F_{n+1}\right)\right) \in \mathbb{N} .
$$

For $k=1, \ldots, \delta$, compute the Newton sums

$$
S_{k}(x):=\operatorname{Res}_{\left(F, F_{n+1}\right)}\left(\operatorname{Jac}\left(F, F_{n+1}\right)\left(s_{n+1} d_{1} \ldots d_{n} f_{0}\right)^{k}\right)
$$

using again Algorithm 2.1.

Step 3. use Newton formulae (2.6) to compute elementary symmetric functions $\sigma_{1}(x), \ldots, \sigma_{\delta}(x)$ of the set $\left\{\frac{f_{0}(\alpha)}{d_{0}(\alpha)} ; \alpha \in \mathcal{Z}_{2}\right\}$.

Step 4. determine the squarefree part $Q\left(x_{0}, \ldots, x_{n}\right)$ of the numerator of the rational function $x_{0}^{\delta}+\sigma_{1}(x) x_{0}^{\delta-1}+\cdots+\sigma_{\delta}(x) \in \mathbb{K}(x)\left[x_{0}\right]$.

OUTPUT: the implicit equation $Q\left(x_{0}, \ldots, x_{n}\right)$ of the rational hypersurface given by the parametrization (3.1). 
Remark 3.1. Changing the order of $x_{0}, \ldots, x_{n}$ in the parametric representation of $(H)$, one may assume that $\operatorname{deg}\left(F_{0}\right)=\max \left(\operatorname{deg}\left(F_{j}\right), j=0, \ldots, n\right)$, thus we get the smallest integers $N$ and $\delta$ possible.

If one denominator $d_{i}$ in the parametric representation of $(H)$ is constant (for instance $d_{0}=1$ ) it is not necessary to add the variable $s_{n+1}$ to compute $S_{k}(x)$ for $k=1, \ldots, \delta:$ in this case, we take $\mathcal{Z}_{2}=\mathcal{Z}$, then $\sigma_{1}(x), \ldots, \sigma_{\delta}(x)$ are the elementary symmetric functions of the set $\left\{f_{0}(\alpha) ; \alpha \in \mathcal{Z}\right\}$ and

$$
S_{k}(x)=\sum_{\alpha \in \mathcal{Z}} f_{0}{ }^{k}(\alpha)=\operatorname{Res}_{\left(F_{1}, \ldots, F_{n}\right)}\left(\operatorname{Jac}\left(F_{1}, \ldots, F_{n}\right){f_{0}}^{k}\right), k=1, \ldots, \delta .
$$

Example 2. We will illustrate our method in order to find the implicit equation of the parametrized surface

$$
x=\frac{t^{2}(s+1)}{s}, y=\frac{s t(s+1)}{s}, z=\frac{t(s+1)}{s} .
$$

This example is taken from [9] ; it is simple but interesting because the moving surface method fails for it (for more details see [9]).

Here, instead of $x_{0}, x_{1}, x_{2}$ (resp. $\left.s_{1}, s_{2}, s_{3}\right)$ we use $x, y, z$ (resp. $\left.s, t, v\right)$.

The polynomials

$$
\begin{aligned}
& F_{0}(s, t)=-t^{2} s-t^{2}+x s \\
& F_{1}(s, t)=-s^{2} t-s t+y s \\
& F_{2}(s, t)=-t s-t+z s \\
& F_{3}(s, t)=v s-1 .
\end{aligned}
$$

To find the implicit equation of this surface we use Algorithm 3.1. So we need to compute Newton sums $S_{k}(y, z)$ by means of Algorithm 2.1. Using Remark 2.2 , we introduce the perturbed system

$$
\begin{aligned}
\Phi_{1}(\lambda, u, s, t) & =\lambda t^{4}+F_{1}-u_{1} \\
\Phi_{2}(\lambda, u, s, t) & =\lambda s^{2}+F_{2}-u_{2} \\
\Phi_{3}(\lambda, u, s, t, v) & =\lambda v^{2}+F_{3}-u_{3} .
\end{aligned}
$$

The polynomials

$$
\begin{aligned}
A_{1}\left(s, u_{1}, u_{2}, u_{3}\right)= & \mathcal{N}_{1}\left(0, u_{1}, u_{2}, u_{3}, s\right)=-s^{2} z+u_{2} s+y s-u_{1}, \\
A_{2}\left(t, u_{1}, u_{2}, u_{3}\right)= & \mathcal{N}_{2}\left(0, u_{1}, u_{2}, u_{3}, t\right)=-t^{2} y-t^{2} z-t^{2} u_{2}-t^{2} u_{1} \\
& +z t y-z t u_{2}+2 u_{1} z t-t u_{2} y-t u_{2}^{2}+u_{2} y z-z^{2} u_{1}, \\
A_{3}\left(v, u_{1}, u_{2}, u_{3}\right)= & \mathcal{N}_{3}\left(0, u_{1}, u_{2}, u_{3}, v\right) \\
= & z u_{3}^{2}-u_{3} y v-u_{3} u_{2} v+2 u_{3} z+v^{2} u_{1}-y v-u_{2} v+z,
\end{aligned}
$$


satisfy the following algebraic relations

$$
A_{1}\left(s, F_{1}, F_{2}\right)=0, A_{2}\left(t, F_{1}, F_{2}\right)=0, A_{3}\left(v, F_{1}, F_{2}, F_{3}\right)=0 .
$$

We deduce from this that the univariate polynomials

$$
\begin{aligned}
& R_{1}(s)=A_{1}(s, 0,0,0)=-s^{2} z+y s, \\
& R_{2}(t)=A_{2}(t, 0,0,0)=-t^{2} y-t^{2} z+z t y, \\
& R_{3}(v)=A_{3}(v, 0,0,0)=-y v+z,
\end{aligned}
$$

belong to the ideal of $(\mathbb{K}[y, z])[s, t, v]$ generated by $F_{1}, F_{2}, F_{3}$. So by the Transformation Law, formulae (2.3) and (2.2), the degree

$$
\delta=\operatorname{Res}_{\left(F_{1}, F_{2}, F_{3}\right)}\left(\operatorname{Jac}\left(F_{1}, F_{2}, F_{3}\right)\right)=1,
$$

and the power sum

$$
S_{1}=\operatorname{Res}_{\left(F_{1}, F_{2}, F_{3}\right)}\left(\operatorname{Jac}\left(F_{1}, F_{2}, F_{3}\right) v s t^{2}(s+1)\right)=-\frac{y z^{2}}{(y+z)} .
$$

The implicit equation of the parametrized surface (3.4) is $-y z^{2}+x z+y x$.

Example 3. We will compute the implicit equation of a surface of bidegree $(1,2)$ studied in [19] :

$$
x=(s-2)(t-3)^{2}, y=s t^{2}, z=(s-1)(t-1)^{2} .
$$

We have

$$
\begin{aligned}
& F_{1}(s, t)=-s t^{2}+y \\
& F_{2}(s, t)=-s t^{2}+2 s t-s+t^{2}-2 t+1+z .
\end{aligned}
$$

Using again Remark 2.2, we introduce the auxiliary system

$$
\begin{aligned}
& \Phi_{1}(\lambda, u, s, t)=\lambda s^{4}-s t^{2}+y-u_{2} \\
& \Phi_{2}(\lambda, u, s, t)=\lambda t^{3}-s t^{2}+2 s t-s+t^{2}-2 t+1+z-u_{3} .
\end{aligned}
$$

We use the same notations as in the previous example. In this case, the univariate polynomials

$$
\begin{aligned}
R_{1}(s) & =s^{4}+(-2-2 y-2 z) s^{3}+\left(z^{2}+2 z+1+y^{2}-2 z y+4 y\right) s^{2} \\
& +\left(2 z y-2 y^{2}-2 y\right) s+y^{2} \\
R_{2}(t) & =t^{4}-2 t^{3}+(1+z-y) t^{2}+2 y t-y
\end{aligned}
$$


We deduce from the fact that the elements $R_{1}(s)$ and $R_{2}(t)$ belong to the ideal generated by $F_{1}$ and $F_{2}$, and the Transformation Law that the degree $\delta=\operatorname{Res}_{\left(F_{1}, F_{2}\right)}\left(\operatorname{Jac}\left(F_{1}, F_{2}\right)\right)$ of the implicit equation that we are looking for and Newton sums $S_{i}=\operatorname{Res}_{\left(F_{1}, F_{2}\right)}\left(\operatorname{Jac}\left(F_{1}, F_{2}\right)(s-2)^{i}(t-3)^{2 i}\right), i=1,2,3,4$, are equal to

$$
\begin{aligned}
\delta= & 4 \\
S_{1}= & -34+6 y+22 z, \\
S_{2}= & 34 y^{2}-516 z+170 z^{2}+290-20 y+424 z y, \\
S_{3}= & -1038 y^{2}-5334 z^{2}-2482+9696 y z^{2}-894 y+1474 z^{3}+4332 z y^{2} \\
& -10242 z y+8214 z+126 y^{3}, \\
S_{4}= & 21314+114572 z^{2}+181488 z^{2} y^{2}+158144 z y+13154 z^{4}-148776 z y^{2} \\
& +23628 y^{2}+32240 z y^{3}+163232 z^{3} y-109832 z-338776 y z^{2}+514 y^{4} \\
& +24440 y-56072 z^{3}-3592 y^{3} .
\end{aligned}
$$

By means of Newton formulae, we obtain the implicit equation of the surface (3.5) which is the following equation of degree 4 :

$$
\begin{aligned}
& 5184+2448 x-6336 y-3492 z x-1960 x y+433 x^{2}-1074 y z x+2512 y^{2} \\
& -5760 z y-80 z x^{2} y-3492 y z^{2}-194 y x^{2}-352 y^{3}+16 y^{4}-194 z y^{2} x+ \\
& 1960 z y^{2}+433 z^{2} y^{2}-136 z y^{3}+320 x y^{2}+x^{2} y^{2}+24 x y^{3}-612 z^{3} y+ \\
& 490 z^{2} x y-6 x^{3} y+1944 z^{3}+34 x^{3}-490 z x^{2}+1440 z^{2} x+x^{4}+157 x^{2} z^{2} \\
& -22 x^{3} z+324 z^{4}-396 x z^{3}+324 z^{2}-7776 z .
\end{aligned}
$$

\section{§3.2. Singular locus}

A singular point of the hypersurface given by the parametrization (3.1) is a common root of $Q$ and its partial derivatives $\frac{\partial Q}{\partial x_{0}}, \ldots, \frac{\partial Q}{\partial x_{n}}$, where $Q$ is the implicit equation of this hypersurface.

For example the singular locus of the surface given in Example 3 can be obtained by the substitution of the parametric representation of the surface in the partial derivatives of the implicit equation, and the computation of the great common divisor of three polynomials in two variables $s$ and $t$. The result is the following implicit curve in the space of parameters $s$ and $t$ surface :

$$
784 t^{2}-580 s t^{2}+96 s^{2} t^{2}-1344 t+1896 s t-672 s^{2} t+576-1740 s+1176 s^{2} .
$$




\section{§3.3. Intersection problem}

Let $\left(H_{1}\right)$ and $\left(H_{2}\right)$ be two hypersurfaces in $\mathbb{C}^{n+1}$ given parametrically. We want to find the intersection of these hypersurfaces. It is easy to obtain it if we implicitize one hypersurface, say $\left(H_{1}\right)$, and we substitute the parametric representation of $\left(\mathrm{H}_{2}\right)$ in the implicit equation of $\left(H_{1}\right)$.

For instance we can compute the intersection of the two surfaces in Examples 2 and 3 . The resulting curve in the space of parameters of the surface (3.5) is the following :

$$
\begin{array}{r}
18+2 t^{4}-16 t^{3}-85 s t^{2}+9 s^{2}+33 s^{2} t^{2}-22 s^{2} t^{3}+14 s^{2} t^{4}+4 s t^{5}-s t^{6}- \\
8 s^{2} t^{5}+2 s^{2} t^{6}-s^{3} t^{2}+4 s^{3} t^{3}-6 s^{3} t^{4}+4 s^{3} t^{5}-s^{3} t^{6}+72 s t+40 s t^{3}- \\
11 s t^{4}-24 s^{2} t-27 s-48 t+44 t^{2}=0 .
\end{array}
$$

\section{$\S 4$. Conclusion}

Other problems in Computer Aided Geometric Design such as the construction of offsets of a given surface, drafts of a surface, ... could be solved thanks to the residue calculus developed in Section 2. This method could be also used to solve some problems in Effective Algebraic Geometry (see [21]).

\section{Acknowledgements}

The first author was supported by the european project GAIA II (IST2001-35512).

\section{References}

[1] I. A. Aizenberg and A. P. Yuzhakov, Integral representations and residues in multidimensional complex analysis, Translated from the Russian by H. H. McFaden, Translation edited by Lev J. Leifman, Amer. Math. Soc., Providence, RI, 1983.

[2] V. Arnold, A. Varchenko and S. Goussein-Zade, Singularities of differential maps, the classification of critical points, caustics and wave fronts, vol. 82, Birkhäuser-Verlag, 1982.

[3] E. Becker, J. Cardinal, M. Roy and Z. Szafraniec, Multivariate Bezoutians, Kronecker symbol and Eisenbud-Levin formula, in Algorithms in Algebraic Geometry and Applications, L. González-Vega and T. Recio, eds., vol. 143 of Progr. Math., Birkhäuser, Basel, 1996, pp. 79-104.

[4] C. A. Berenstein, R. Gay, A. Vidras and A. Yger, Residue Currents and Bezout Identities, vol. 114 of Progr. Math., Birkhäuser, 1993.

[5] C. A. Berenstein and A. Yger, Effective Bezout identities in $\mathbb{Q}\left[z_{1}, \cdots, z_{n}\right]$, Acta Math. 166 (1991), no. 1-2, 69-120.

[6] $\longrightarrow$, Residue calculus and effective Nullstellensatz, Amer. J. Math. 121 (1999), no. 4, 723-796. 
[7] B. Buchberger, Applications of Gröbner bases in nonlinear computational geometry, in Trends in computer algebra (Bad Neuenahr, 1987), 52-80, Lecture Notes in Comput. Sci., 296, Springer, Berlin.

[8] L. Busé, Residual resultant over the projective plane and the implicitization problem, in Proceedings of the 2001 International Symposium on Symbolic and Algebraic Computation, 48-55 (electronic), ACM, New York.

[9] L. Busé, D. Cox and C. D'Andrea, Implicitization of surfaces in $\mathbb{P}^{3}$ in the presence of base points, J. Algebra Appl. 2 (2003), no. 2, 189-214.

[10] L. Busé and J.-P. Jouanolou, On the closed image of a rational map and the implicitization problem, J. Algebra 265 (2003), no. 1, 312-357.

[11] D. Manocha and J. F. Canny, Implicit representation of rational parametric surfaces, J. Symbolic Comput. 13 (1992), no. 5, 485-510.

[12] E. Cattani, A. Dickenstein and B. Sturmfels, Computing multidimensional residues, in Algorithms in algebraic geometry and applications (Santander, 1994), 135-164, Progr. Math., 143, Birkhäuser, Basel.

[13] R. M. Corless, M. Giesbrecht, I. Kotsireas and S. Watt, Numerical implicitization of parametric hypersurfaces with linear algebra, in Artificial intelligence and symbolic computation (Madrid, 2000), 174-183, Lecture Notes in Comput. Sci., 1930, Springer, Berlin.

[14] D. Cox, R. Goldman and M. Zhang, On the validity of implicitization by moving quadrics of rational surfaces with no base points, J. Symbolic Comput. 29 (2000), no. 3, 419-440.

[15] A. Dickenstein and C. Sessa, An effective residual criterion for the membership problem in $\mathbb{C}\left[z_{1}, \cdots, z_{n}\right]$, J. Pure Appl. Algebra 74 (1991), no. 2, 149-158.

[16] T. Dokken, Approximate implicitization, in Mathematical methods for curves and surfaces (Oslo, 2000), 81-102, Vanderbilt Univ. Press, Nashville, TN.

[17] M. Elkadi, Bornes pour les degrés et les hauteurs dans le problème de division, Michigan Math. J. 40 (1993), no. 3, 609-618.

[18] — Résidu de Grothendieck et forme de Chow, Publ. Mat. 38 (1994), no. 2, 381-393.

[19] M. Elkadi, A. Galligo and T. H. Lê, Parametrized surfaces in $\mathbb{P}^{3}$ of bidegree $(1,2)$, in ISSAC 2004, 141-148, ACM, New York.

[20] M. Elkadi and B. Mourrain, Algorithms for residues and Lojasiewicz exponents, J. Pure Appl. Algebra 153 (2000), no. 1, 27-44.

[21] - Introduction à la résolution des systèmes polynomiaux, to appear, 2006.

[22] - Residue and implicitization problem for rational surfaces, Appl. Algebra Engrg. Comm. Comput. 14 (2004), no. 5, 361-379.

[23] N. Fitchas, M. Giusti and F. Smietanski, Sur la complexité du théorème des zéros, in Approximation and optimization in the Caribbean, II (Havana, 1993), 274-329, Lang, Frankfurt am Main.

[24] L. Gonzalez-Vega, Implicitization of parametric curves and surfaces by using multidimensional Newton formulae, J. Symbolic Comput. 23 (1997), no. 2-3, 137-151.

[25] T. Krick, L. M. Pardo and M. Sombra, Sharp estimates for the arithmetic Nullstellensatz, Duke Math. J. 109 (2001), no. 3, 521-598.

[26] E. Kunz, Kähler differentials, Vieweg, Braunschweig, 1986.

[27] H. Matsumura, Commutative algebra, Second edition, Benjamin/Cummings Publishing Co., Inc., Reading, Mass., 1980.

[28] G. Scheja and U. Storch, Über Spurfunktionen bei vollständigen Durchschnitten, J. Reine Angew. Math. 278/279 (1975), 174-190.

[29] T. Sederberg, D. Anderson and R. Goldman, Implicit representation of parametric curves and surfaces, Computer Vision, Graphics and Image Processing, 28 (1984), pp. $72-84$.

[30] T. Sederberg and F. Chen, Implicitzing using moving curves and surfaces, in proceedings of SIGGRAPH, 1995, pp. 301-308.

[31] A. Yuzhakov, On the computation of the complete sum of residues relative to a polynomial mapping in $\mathbb{C}^{n}$, Sov. Math., Dokl., 29 (1984), pp. 321-324. 